\title{
COHOMOLOGICAL FINITENESS CONDITIONS FOR ELEMENTARY AMENABLE GROUPS
}

\author{
P. H. KROPHOLLER, C. MARTÍNEZ-PÉREZ, AND B. E. A. NUCINKIS
}

\begin{abstract}
It is proved that every elementary amenable group of type $\mathrm{FP}_{\infty}$ admits a cocompact classifying space for proper actions.
\end{abstract}

\section{INTRODUCTION}

For discrete groups the term cohomological finiteness condition refers to any property of groups which holds for all groups that admit finite Eilenberg-Mac Lane spaces. Amongst such properties there are classical finiteness conditions such as finite generation and finite presentability and there are also the more exotic conditions such as type $\mathrm{FP}_{\infty}$, type FP, and type FL. Note also that the property of being torsion-free is a cohomological finiteness condition but not a classical finiteness condition while residual finiteness is a classical finiteness condition but not a cohomological finiteness condition.

In recent years there has been increasing interest in a variant of the EilenbergMac Lane space, namely the classifying space for proper actions. In this paper we shall determine the precise conditions under which elementary amenable groups admit cocompact proper classifying spaces. Bredon cohomology plays a role in studying these classifying spaces in a way that runs largely parallel to the role of ordinary group cohomology in studying Eilenberg-Mac Lane spaces and their universal covers. In Bredon cohomology, the group $G$ is replaced by the orbit category $\mathscr{O}_{\mathscr{X}} G$ defined with respect to a suitable family of subgroups $\mathscr{X}$. In this paper we shall only be concerned with the family $\mathscr{F}$ of finite subgroups and so we simply write $\mathscr{O} G$ for the orbit category instead of $\mathscr{O}_{\mathscr{F}} G$. Modules over the orbit category are contravariant functors to the category of abelian groups. These are called $\mathscr{O} G$ modules. The category of $\mathscr{O} G$-modules has enough projectives and homological algebra can be developed using projective resolutions giving rise to the Bredon cohomology of groups with clear resemblance to ordinary group cohomology.

All the notions type $\mathrm{FP}_{\infty}$, type $\mathrm{FP}$, type $\mathrm{FL}$, etc have analogues when considering Bredon projective resolutions and we refer to these by the names type Bredon $\mathrm{FP}_{\infty}$, type Bredon FP, type Bredon FL, etc. Figure 1 shows the various interrelationships

2000 Mathematics Subject Classification. 57Q05, 20 J05.

Key words and phrases. Bredon cohomology, elementary amenable group, proper classifying space, Eilenberg-Mac Lane space, finite type.

The second named author was partially supported by MTM2004-08219-C02-01 and Gobierno de Aragón. 


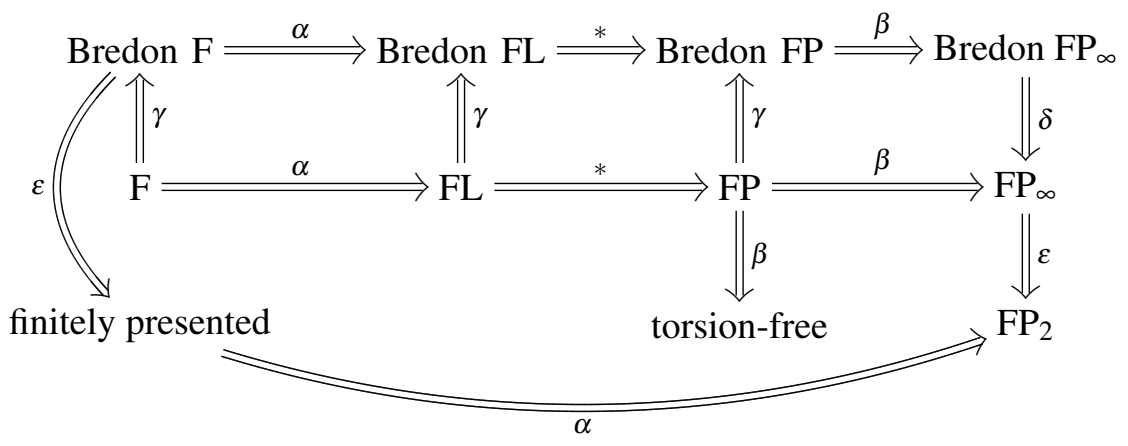

FIGURE 1. Connections between cohomological finiteness conditions

between these properties and all these implications are easily established. The notion of type Bredon $\mathrm{F}$ is by definition equivalent to the existence of a cocompact proper classifying space. Save for the two implications marked $*$ all the implications shown are known to be irreversible. We have included a short explanation of this diagram in the last section of this paper.

For virtually soluble groups or, more generally, elementary amenable groups there are no surprises as to which groups are of Bredon type $\mathrm{F}$ and our main theorem is as follows.

Theorem 1.1. Let $G$ be an elementary amenable group. Then the following are equivalent.

(i) $G$ is of type Bredon $\mathrm{F}$.

(ii) $G$ is of type Bredon FL.

(iii) $G$ is of type Bredon FP.

(iv) $G$ is of type Bredon $\mathrm{FP}_{\infty}$.

(v) $G$ is virtually of type $\mathrm{F}$.

(vi) $G$ is virtually of type $\mathrm{FL}$.

(vii) $G$ is virtually of type FP.

(viii) $G$ is of type $\mathrm{FP}_{\infty}$.

(ix) $G$ is constructible.

(x) Either $G$ is polycyclic-by-finite or $G$ has a normal subgroup $K$ such that $G / K$ is a Euclidean crystallographic group and for each subgroup $L \supseteq K$ with $L / K$ finite there is a finitely generated virtually nilpotent subgroup $B=B(L)$ of $L$ and an element $t=t(L)$ of $L$ such that $t^{-1} B t \subset B$ and $L=B *_{B, t}$ is a strictly ascending $H N N$-extension with base $B$ and stable lettert.

Here (ix) refers to the notion of constructible group as introduced by Baumslag and Bieri [3]. The class of constructible groups is the smallest class of groups closed under forming amalgamated free products, HNN-extensions and finite extensions and all such groups are finitely presented and of type $\mathrm{FP}_{\infty}$. Baumslag and Bieri provide a thorough discussion of the nature of soluble constructible groups, [3]. Further analysis of this class appears in the work [11] of Bieri and Strebel and plays a crucial role in this paper.

In view of known interconnections between the conditions (i)-(x), which we discuss below, most of the work in this paper is concerned with establishing (viii) 
$\Rightarrow(\mathrm{x}) \Rightarrow(\mathrm{i})$. The first of these implications is the subject of $\S 2$ and the second is the subject of $\S 4$. For the second implication we use Bredon cohomology which we review in $\S 3$.

The following is an immediate consequence. Note that prior to this work this Corollary was not proven even for the class of soluble groups of type FP.

Corollary 1.2. Every elementary amenable group of type $\mathrm{FP}$ is of type $\mathrm{F}$.

Proof. Let $G$ be an elementary amenable group of type FP. According to Theorem 1.1. $G$ is of type Bredon F and so admits a cocompact model for the classifying space for proper actions $\underline{E} G$. Since every group of type FP, in particular $G$, is torsion free it follows that $\underline{E} G=E G$ and $G$ is of type $\mathrm{F}$.

Corollary 1.3. Let $G$ be an elementary amenable group of type Bredon $\mathrm{F}$ and let $\Phi$ be a finite group of automorphisms of $G$. Then the subgroup $\operatorname{Fix}(\Phi)$ of elements of $G$ fixed by every element of $\Phi$ is also of type Bredon $\mathrm{F}$.

Proof. The split extension, or semidirect product, $G \rtimes \Phi$ is elementary amenable and of type $\mathrm{FP}_{\infty}$ since both of these properties are inherited by finite index over groups. Therefore, according to Theorem 1.1 (viii) $\Rightarrow$ (i), $G \rtimes \Phi$ is of type F. Normalizers of finite subgroups of groups of type $F$ are always of type $F$ and hence the normalizer $N_{G \rtimes \Phi}(\Phi)$ is of type F. The subgroup Fix $(\Phi)$ has finite index in this normalizer and so is also of type $\mathrm{F}$.

For any group $G$ satisfying the conditions in the Theorem one has

$$
\mathrm{h} G=\operatorname{cd}_{\mathbb{Q}} G=\underline{\operatorname{cd}} G<\infty
$$

where $\mathrm{h} G$ is the Hirsh rank of $G$ and $\underline{\mathrm{cd}} G$ the Bredon cohomological dimension, which is the analogous of the usual cohomological dimension but for Bredon cohomology. This leads to the following:

Conjecture 1.4. The conditions

(xi) $\mathrm{h} G=\operatorname{cd}_{\mathbb{Q}} G<\infty$

(xii) $\mathrm{h} G=\underline{\mathrm{cd}} G<\infty$

can be added to the Theorem.

It follows from [12] that for any group $\operatorname{cd}_{\mathbb{Q}} G \leq \underline{\operatorname{cd}} G$ and hence (xii) implies (xi) in the above conjecture. Furthermore (xi) implies that $G / T$ is of type Bredon F, where $T$ is the largest normal locally finite subgroup of $G$. Hence a positive answer to the following would also prove Conjecture 1.4 .

Conjecture 1.5. Let $G$ be an elementary amenable group such that $\mathrm{h} G=\operatorname{cd}_{\mathbb{Q}} G$ is finite. Then $G$ has a bound on the orders of the finite subgroups.

Background to the Theorem. Historically, the very first steps towards understanding cohomological finiteness conditions for soluble groups were taken by Gruenberg and Stammbach. Key steps concerning nilpotent groups appear in Gruenberg's notes [18] and homological dimension was computed by Stammbach [32]. The question was further investigated and highlighted by Bieri [6]. Gildenhuys determined exactly which soluble groups have cohomological dimension 2: the solution [15] shows that all non-abelian such groups are ascending HNN-extensions of type $\mathrm{F}$ and is the first evidence that questions about cohomological finiteness conditions for soluble groups would prove to be substantial and interesting. For 
soluble-by-finite groups, the equivalence of conditions (vi), (vii) and (ix) of Theorem 1.1 was established by Gildenhuys, Strebel and Kropholler, [16, 17, 20]. Subsequently it was shown [21, 22] by Kropholler that soluble groups of type $\mathrm{FP}_{\infty}$ are virtually of type FP and work [19] of Hillman and Linnell made it possible to extend the results to the elementary amenable case. At this stage it became clear that every elementary amenable group of type $\mathrm{FP}_{\infty}$ is nilpotent-by-abelian-byfinite, constructible and virtually of type F. However it remained an open problem whether or not elementary amenable groups, or even soluble groups, of type FP are necessarily of type FL. Moreover, the interest in proper classifying spaces, which are natural to consider for groups with torsion, raised questions as to whether all soluble groups of type $\mathrm{FP}_{\infty}$ satisfied the strongest Bredon finiteness conditions, see for example [27] where the equivalence between (viii) and (iii) is proven. Theorem 1.1 shows that this is the case. Part (x) comes about through a careful analysis of the Bieri-Strebel strategy for characterizing properties of nilpotent-by-abelian-byfinite groups using invariants [11], commonly called BNS-invariants [7], which are subsets of certain valuation spheres.

\section{BIERI-STREBEL INVARIANTS FOR NILPOTENT-BY-ABELIAN-BY-FINITE GROUPS, AND THE PROOF OF THEOREM 1.1 (viii) $\Rightarrow(\mathrm{x})$}

The goal of this section is to establish the more refined structure theory for $G$ stated in Theorem 1.1 (x). As explained above, results in [21, 22] and in [19] imply that any elementary amenable group of type $\mathrm{FP}_{\infty}$ is finite-by-virtually soluble. By taking the centralizer of the finite normal subgroup, which is soluble of finite index one easily sees that the group is in fact nilpotent-by-abelian-by-finite.

We adopt the notation used by Bieri and Strebel in [11]. For a finitely generated abelian group $Q$ we write $S(Q)$ for the valuation sphere as defined in $\S 1.1$ of [11]. Let $(N, H)$ be an admissible pair of subgroups of the group $G$, meaning that the following conditions are satisfied:

- $N$ and $H$ are both normal subgroups of $G$;

- $N \subseteq H$;

- $N$ is nilpotent;

- $H / N$ is abelian; and

- $G / H$ is finite.

Now let $P$ denote the largest normal locally polycyclic subgroup of $H$. Then $H / P$ is a finitely generated abelian group and so the valuation sphere $S(H / P)$ is defined. Moreover, Bieri and Strebel show that if $\left(N^{\prime}, H^{\prime}\right)$ is another admissible pair then the valuations spheres $S(H / P)$ and $S\left(H^{\prime} / P^{\prime}\right)$ can be identified in a canonical way. Therefore they define the valuation sphere $S(G)$ to be $S(H / P)$ for some fixed choice of admissible pair $(N, H)$ without any essential ambiguity.

Bieri and Strebel introduce the invariant $\sigma(G)$, a certain closed subset of $S(G)$. We shall be interested in the following results about this invariant which are the content of Theorems 5.2 and 5.4 of [11].

\section{Proposition 2.1.}

(i) $G$ is constructible if and only if $\sigma(G)$ is contained in an open hemisphere.

(ii) $G$ is polycyclic-by-finite if and only if $\sigma(G)$ is empty.

Taking $Q$ to be the quotient group $H / N$ we naturally have that $S(G)=S(H / P)$ is a subsphere of $S(Q)$. 
We now note that the action of $G$ by conjugation on $H$ stabilizes $\sigma(G)$ (see [27, Lemma 3.4]). Moreover it induces an action of the finite group $G / H$ on both $H / N$ and $H / P$. In turn this induces actions of $G / H$ on the vector spaces hom $(H / P, \mathbb{R})$ and $\operatorname{hom}(H / N, \mathbb{R})$ and hence also on the valuation spheres $S(G)=S(H / P)$ and $S(H / N)$ stabilizing $\sigma(G)$.

Also, the group $G$ acts by conjugation on $N$ and this passes to an action of $G / N$ on the largest abelian quotient $N_{\mathrm{ab}}=N /[N, N]$ of $N$. In this way we may view $N_{\mathrm{ab}}$ as a right $\mathbb{Z}[G / N]$-module. For $x \in G$ and $b \in N$ we write $b^{x}$ for the conjugate $x^{-1} b x$ and we write $\bar{x}$ for the image of $x$ in $G / N$, that is $\bar{x}$ is the coset $N x$. If $a$ is an element of $N_{\mathrm{ab}}$ so that is $a=[N, N] b$ for some $b \in N$ then we write $a \bar{x}$ for the coset $[N, N] b^{x}$. In other words we shall notate $N_{\mathrm{ab}}$ as a right $G / N$-module.

$\mathscr{Q}$ of $Q$ and a finitely generated subgroup $B_{0}$ of $N_{\mathrm{ab}}$

Associated to the data $G, H, N$ we may consider the following subsets $\mathscr{B}_{0}$ and $\mathscr{B}$ of $G$.

- $\mathscr{B}_{0}$ is defined to be the set of $x$ in $G$ for which there exists a finitely generated subgroup $B_{0}$ of $N_{\mathrm{ab}}$ such that $B_{0} \bar{x} \subseteq B_{0}$ and $\bigcup_{i \leq 0} B_{0} \bar{x}^{i}=N_{\mathrm{ab}}$.

- $\mathscr{B}$ is defined to be the set of $x$ in $G$ for which there exists a finitely generated subgroup $B$ of $N$ such that $B^{x} \subseteq B$ and $\bigcup_{i \leq 0} B^{x^{i}}=N$.

We shall now make the assumption that $G$ is constructible. Therefore by Proposition 2.1 (i) the invariant $\sigma(G)$ is contained in an open hemisphere of $S(G)$ and the following three lemmas all rely on this. We shall also assume that $\sigma(G)$ is non-empty, i.e., that $G$ is not polycyclic-by-finite.

Lemma 2.2. There exists $x \in \mathscr{B}_{0}$ whose image $\bar{x}$ in $G / N$ belongs to the centre $\zeta(G / N)$.

Proof. As remarked above the finite group $G / H$ acts on $Q$ and on the sphere $S(Q)$ stabilizing $\sigma(G)$. Now let

$$
C / N=C_{H / N}(G / H) \leq \zeta(G / N) .
$$

Since $Q$ is finitely generated abelian [27, Lemma 3.5] implies that by changing $H$ if necessary to a finite index subgroup we may assume that $H / N$ is torsion free and $H / N=C / N \times T / N$ with $T / N$ as in [27, Lemma 3.6]. So applying that result we deduce that $\sigma(C)$ is contained in an open hemisphere. If $\operatorname{rk} C / N=0$ then $\sigma(C)=\varnothing$, and hence $C$ and also $G$ would be polycyclic-by-finite. Therefore by [10, Theorem 4.6] there are elements $q_{1}, \ldots, q_{s}$ of $C / N$ and a finitely generated subgroup $B_{0}$ of $N_{\mathrm{ab}}$ such that $q_{i}$ all satisfy the condition $B_{0} q_{i} \subseteq B_{0}$ and $N_{\mathrm{ab}}=$ $\bigcup_{i<0} B_{0}\left(q_{1} \ldots q_{n}\right)^{i}$. It suffices then to take $x$ with $\bar{x}=q_{1} \ldots q_{s}$.

The previous result can also be proven as follows. Since $\sigma(G)$ is non empty, compact (in fact results of Bieri and Strebel show that it is finite in this case) and stabilized by the finite group $G / H$ it follows that its centre of mass is a fixed point of $G / H$. Then one can argue in a similar way as in [10, Theorem 4.6] but using this fixed element to deduce the existence of a a finite subset $\left\{q_{1}, \ldots, q_{m}\right\}$ of $Q$ with the following properties:

- $\left\{q_{1}, \ldots, q_{m}\right\}$ is invariant under the action of the finite group $G / H$, and $\left\langle q_{1}, \ldots, q_{m}\right\rangle$ generate a subgroup of finite index in $Q$. 
- There is a finitely generated subgroup $B_{0}$ of $N_{\mathrm{ab}}$ such that the $q_{i}$ all satisfy the condition $B_{0} q_{i} \subseteq B_{0}$.

- $N_{\mathrm{ab}}=\bigcup_{i<0} B_{0}\left(q_{1} \ldots q_{m}\right)^{i}$.

Then one takes $x \in G$ with $\bar{x}=q_{1} \ldots q_{m}$.

Lemma 2.3. $\mathscr{B}_{0}=\mathscr{B}$.

Proof. This is an easy variation on the proof of ([11], Theorem 5.2). Take $x \in \mathscr{B}_{0}$ and consider the derived series of $N$ with

$$
\begin{gathered}
\gamma_{1} N=N, \\
\gamma_{i+1} N=\left[\gamma_{i} N, N\right] .
\end{gathered}
$$

We prove by induction on $\ell$ that there is some finitely generated subgroup $B_{\ell+1}$ of $N / \gamma_{\ell+1} N$ with $B_{\ell+1}^{x} \subseteq B_{\ell+1}$ and $\bigcup_{i \leq 0} B_{\ell+1}^{x^{i}}=N$. When $\ell=1$ there is nothing to prove and so we assume that $\ell \geq 2$ and that there exists a finitely generated subgroup $B_{\ell}$ with the desired properties. Let $A_{\ell}$ be a finitely generated subgroup of $N / \gamma_{\ell+1} N$ with $A_{\ell} \gamma_{\ell} N / \gamma_{\ell} N=B_{\ell}$. Exactly as in ([11], Theorem 5.2) one gets a finitely generated subgroup $A_{0}$ of $\gamma_{\ell} N / \gamma_{\ell+1} N$ having similar properties as those of $B_{\ell}$ and it sufices to take

$$
B_{\ell+1}=A_{\ell} A_{0} .
$$

Using Lemma 2.2, choose $x \in \mathscr{B}_{0}$ such that $\bar{x}$ belongs to $\zeta(G / N)$. By Lemma 2.3. $x$ belongs to $\mathscr{B}$ and so we may choose a finitely generated subgroup $B$ of $N$ such that $B^{x} \subseteq B$ and $N=\bigcup_{i<0} B^{x^{i}}$. We now keep $x$ and $B$ fixed for the remainder of this section. Note that if $x$ has finite order then $G$ is polycyclic-by-finite. We shall therefore assume that $x$ has infinite order.

Lemma 2.4. The subgroup $K:=\langle B, x\rangle$ is normal in $G$ and if $L$ is any subgroup containing $K$ such that $L / K$ is finite then there exist $y \in G$ and a finitely generated subgroup $D$ of $L$ such that

(i) $B$ is a subgroup of finite index in $D$,

(ii) $D^{y} \subseteq D$,

(iii) $\bar{y}^{k}=\bar{x}^{\ell}$ for some positive integers $k, \ell$,

(iv) $L=\langle D, y\rangle$.

Proof. Since $N=\bigcup_{i<0} B^{x^{i}}$ we have that $N \subseteq K$ and clearly $K / N$ is the cyclic group generated by $\bar{x}$ which is central in $G / N$. Thus $K$ is normal in $G$.

Suppose now that $L$ is a subgroup of $G$ containing $K$ such that $L / K$ is finite. Then $L / N$ is virtually infinite cyclic and centre-by-finite. Let $N_{1} / N$ denote the largest finite normal subgroup of $L / N$. Then $L / N_{1}$ is infinite cyclic. Choose $y$ to be a generator of $L$ modulo $N_{1}$. Then $\bar{y}$ and $\bar{x}$ generate commensurable cyclic subgroups of $L / N$ and so, replacing $y$ by $y^{-1}$ if necessary, we may assume that there are positive integers $k$ and $\ell$ such that $\bar{y}^{k}=\bar{x}^{\ell}$. Notice that $x^{\ell} y^{-k}$ is then an element of $N$.

Let $\mathscr{N}_{1}$ be a finite subset of $G$ consisting of coset representatives for the elements of $N_{1} / N$. Since $\bar{x}$ is central in $G / N$ it follows that for each $g \in \mathscr{N}_{1}$ there exists 
$n_{g} \in N$ such that $g^{x}=g n_{g}$. Choose $j<0$ so that $B^{x^{j}}$ contains all the elements $n_{g}$ as $g$ runs through $\mathscr{N}_{1}$ and so that it also contains $x^{\ell} y^{-k}$. The choice of $j$ is possible because there are only finite many $n_{g}$ and $N$ is the directed union $\bigcup_{i<0} B^{x^{i}}$. Now consider the group $B_{1}:=\left\langle B^{x^{j}} \cup \mathscr{N}_{1}\right\rangle$. This is contained in the virtually nilpotent group $N_{1}$ and so is itself virtually nilpotent. Also, $B_{1}$ is finitely generated, $B_{1}^{x} \subseteq B_{1}$, and we have $N_{1}=\bigcup_{i<0} B_{1}^{x^{i}}$. Define $D$ by

$$
D:=\left\langle B_{1}, B_{1}^{y}, \ldots, B_{1}^{y^{k-1}}\right\rangle .
$$

Then $D$ has the desired properties. First, $D^{y}$ is generated by $B_{1}^{y}, \ldots, B_{1}^{y^{k-1}}, B_{1}^{y^{k}}$ and since $x^{\ell} y^{-k}$ belongs to $B_{1}$ we have $B_{1}^{y^{k}}=B_{1}^{x^{\ell}} \subseteq B_{1}$ so that $D^{y} \subseteq D$. Secondly $\langle D, y\rangle$ contains $N, \mathscr{N}_{1}$ and $y$ so equals $L$.

Establishing the structure described in Theorem 1.1 $(x)$. There are two cases according to whether or not $\sigma(G)$ is empty. If $\sigma(G)=\varnothing$ then $G$ is polycyclicby-finite by Proposition 2.1 (ii) and we are done. If $\sigma(G) \neq \varnothing$ then Lemma 2.4 applies. In that case, let $K$ be as in Lemma 2.4 and let $K_{1} / K$ be largest finite normal subgroup of $G / K$. Since $G / K$ is a quotient of $G / H$ it is finitely generated and abelian-by-finite. Therefore $G / K_{1}$ is a Euclidean crystallographic group. Lemma 2.4 shows that any overgroup $L$ of finite index over $K$ or $K_{1}$ also enjoys the structure of being an ascending HNN-extension. Therefore we may replace $K$ by $K_{1}$ and have the desired conclusion.

\section{BREDON COHOMOLOGY AND FINITENESS CONDITIONS FOR PROPER CLASSIFYING SPACES}

Let $G$ be a group. We write $\mathscr{O} G$ for the orbit category of $G$ with respect to the class of finite subgroups of $G$. The orbit category has the transitive $G$-sets with finite stabilizers as objects and $G$-maps between them as morphisms. Modules over the orbit category are contravariant functors from the orbit category to the category of abelian groups. A sequence $A \rightarrow B \rightarrow C$ of $\mathscr{O} G$-modules is exact at $B$ if and only if each instance is exact, that is $A(\Delta) \rightarrow B(\Delta) \rightarrow C(\Delta)$ is exact at $B(\Delta)$ for every transitive $G$-set $\Delta$.

For $G$-sets $\Delta, \Omega$ we write $[\Delta, \Omega]_{G}$ for the set of $G$-maps from $\Delta$ to $\Omega$ and we write $\mathbb{Z}[\Delta, \Omega]_{G}$ for the free abelian group on $[\Delta, \Omega]_{G}$. When there is no ambiguity we drop the symbol $G$ and simply write $[\Delta, \Omega]$. Fixing $\Omega$ and allowing $\Delta$ to range over transitive $G$-sets with finite stabilizers we obtain an $\mathscr{O} G$-module $\mathbb{Z}[, \Omega]$. The trivial $\mathscr{O} G$-module, usually written $\underline{\mathbb{Z}}$, arises from this construction by taking $\Omega$ to be the one-point $G$-set. For any finite group $H$, the $\mathscr{O} G$-module $\mathbb{Z}[, H \backslash G]$ is projective and direct sums of modules of this form (allowing different finite subgroups) are called free $\mathscr{O} G$-modules. Every projective $\mathscr{O} G$-module is a direct summand of a free module and the finitely generated projective modules are precisely the direct summands of finite direct sums of modules of the form $\mathbb{Z}[, H \backslash G]$ with $H$ finite. The notions of type Bredon FP, Bredon FL, Bredon FP ${ }_{\infty}$ are defined in terms of projective resolutions of $\underline{\mathbb{Z}}$ over $\mathscr{O} G$ in just the same way that the classical notions of type FP, FL and $\mathrm{FP}_{\infty}$ are defined. 
Moreover it is also possible to define the notion of type Bredon $\mathrm{FP}_{n}$ for each $n \geq 0$. The following Lemma implies the Bredon analogue of the classical fact that a group is of type $\mathrm{FP}_{1}$ if and only if it is finitely generated.

Lemma 3.1. Let $G$ be a group. Then $G$ is of type Bredon $\mathrm{FP}_{0}$ if and only if $G$ has only finitely many conjugacy classes of finite subgroups, and $G$ is of type Bredon $\mathrm{FP}_{n}$ if and only if, in addition, the Weyl-group $K \backslash N_{G}(K)$ of each finite subgroup $K$ is of type $\mathrm{FP}_{n}$.

Proof. If $G$ is of type Bredon $\mathrm{FP}_{0}$ then there is a $G$-finite $G$-set $\Omega$ with finite stabilizers and an epimorphism

$$
\mathbb{Z}[, \Omega] \rightarrow \underline{\mathbb{Z}} .
$$

Now let $K$ be an arbitrary finite subgroup of $G$. Evaluating this epimorphism at $\Omega$ we obtain an epimorphism $\mathbb{Z} \Omega^{K} \rightarrow \mathbb{Z}$ and therefore $\Omega^{K}$ is non-empty. This shows that $K$ belongs to the finite set of conjugacy classes of subgroups which have fixed points in $\Omega$. Conversely, if there are only finitely many conjugacy classes of finite subgroups then we can take $\Omega=\bigsqcup_{H} H \backslash G$ where $H$ runs through a set of conjugacy class representatives of finite subgroups, and the obvious augmentation $\operatorname{map} \mathbb{Z}[, \Omega] \rightarrow \mathbb{Z}$ is an epimorphism.

The necessary and sufficient conditions for Bredon type $\mathrm{FP}_{n}$ are consequences of the following lemma.

We say a Bredon module $M$ is finitely generated if there is a finite $\mathscr{O} G$-set $\Sigma$ in the sense of Lück [24, 9.16, 9.19] such that there is a free Bredon module $F$ on $\Sigma$ mapping onto $M$. An $\mathscr{O} G$-set $\Sigma$ is determined by sets $\Sigma_{K}$ for each finite subgroup $K$. $\Sigma$ is said to be finite if for all finite subgroups $K, \Sigma_{K}$ is finite and $\Sigma_{H}=\varnothing$ for all but finitely many finite subgroups $H$.

Lemma 3.2. Let $G$ be a group with finitely many conjugacy classes of finite subgroups. Then a Bredon module $M$ is of type Bredon $\mathrm{FP}_{n}$ if and only if for each finite subgroup $K$ of $G, M(K \backslash G)$ is a module of type $\mathrm{FP}_{n}$ over the Weyl-group $W K=K \backslash N_{G}(K)$.

Proof. Let $M$ be a Bredon module of type Bredon $\mathrm{FP}_{n}$ and let $P_{*} \rightarrow M$ be a projective resolution. We may assume that all $P_{i}$ for $i \leq n$ are finitely generated free Bredon modules. An argument analogous to [29, Section 3] shows that upon evaluating the $P_{i}(K \backslash G)$ are finitely generated permutation modules over $W K$ with finite stabilizers. Hence, by [28, Proposition 6.3], $P_{i}(K \backslash G)$ is of type $\mathrm{FP}_{\infty}$ for all $i \leq n$. A dimension shift, see [6, Proposition 1.4], implies that $M(K \backslash G)$ is a $W K$-module of type $\mathrm{FP}_{n}$.

The converse is proved by induction on $n$. Let $n=0$ and $M$ be a Bredon module such that $M(K \backslash G)$ is a finitely generated $W K$-module for all finite subgroups $K$. We will construct a finite $\mathscr{O} G$-set $\Sigma$ generating $M$ as a Bredon module. The free Bredon module on $\Sigma$ then is finitely generated and maps onto $M$. Recall that for every $G$-map $H \backslash G \rightarrow K \backslash G$ there is a homomorphism of abelian groups $\varphi_{K}^{H}: M(K \backslash G) \rightarrow M(H \backslash G)$. For each finite subgroup $H$ of $G$ fix a finite generating set $X_{H}$ of $M(H \backslash G)$. Now $\Sigma_{H}$ is the union of $X_{H}$ with all elements of $M(H \backslash G)$ of the form $\varphi_{K}^{H}\left(x_{K}\right)$ whenever there is a $G$-map $H \backslash G \rightarrow K \backslash G$ and $x_{K} \in X_{K}$. Since there are only finitely many conjugacy classes of finite subgroups this results in a finite set $\Sigma_{H}$. Since only one representative for each finite subgroup needs to be 
taken into account, the resulting $\mathscr{O} G$-set is indeed finite. There are maps $\Sigma_{K} \rightarrow \Sigma_{H}$ induced by the maps $\varphi_{K}^{H}$ and the free module $F$ on $\Sigma$ maps onto $M$.

Now suppose $n>0$ and the claim is true for $k<n$. Since for each finite subgroup $M(K \backslash G)$ is a $W K$-module of type $\mathrm{FP}_{n}$ it is in particular finitely generated. And we have shown that there is a short exact sequence of Bredon modules $K_{0} \longmapsto P_{0} \rightarrow M$ with $P_{0}$ finitely generated free. Then, as above, $P_{0}(H \backslash G)$ is a $W H$-module of type $\mathrm{FP}_{\infty}$. Hence by [6, Proposition 1.4] all $K_{0}(H \backslash G)$ are $W H$-modules of type $\mathrm{FP}_{n-1}$ for all finite subgroups $H$ of $G$. By induction $K_{0}$ is of type Bredon $\mathrm{FP}_{n-1}$ and the claim follows.

Let $X$ be a $G$-complex in the sense of tom Dieck ([34], Chapter II): this is a $G$-CW-complex on which $G$ acts by permuting the cells and in such a way that the stabilizer of each cell fixes that cell point by point. We shall write $\Delta^{n}(X)$ for the set of $n$-cells of $X$.

Lemma 3.3. Let $X$ be a $G$-complex such that the fixed sets $X^{H}$ are acyclic for all finite subgroups of $G$. Then the augmented Bredon cell complex

$\cdots \rightarrow \mathbb{Z}\left[\quad, \Delta^{n}(X)\right] \rightarrow \mathbb{Z}\left[\quad, \Delta^{n-1}(X)\right] \rightarrow \cdots \rightarrow \mathbb{Z}\left[\quad, \Delta^{1}(X)\right] \rightarrow \mathbb{Z}\left[\quad, \Delta^{0}(X)\right] \rightarrow \underline{\mathbb{Z}} \rightarrow 0$

is an exact sequence of $\mathscr{O} G$-modules.

Proof. To check that the Bredon cell complex is exact it suffices to check that the chain complex obtained by evaluating on each transitive $G$-set with finite stabilizers is exact. A typical such $G$-set has the form $H \backslash G$, that is the set of right cosets of some finite subgroup $H$. For any $G$-set $\Omega$ the set $[H \backslash G, \Omega]$ can be identified with the $H$-fixed point set $\Omega^{H}$ and so when we evaluate the Bredon complex at $H \backslash G$, what we see is the ordinary augmented cellular chain complex of the space $X^{H}$. Thus the lemma follows from the assumption that all the fixed sets $X^{H}$ are acyclic.

Corollary 3.4. If $T$ is a $G$-tree with edge set $E$ and vertex set $V$ then the augmented Bredon cell complex

$$
0 \rightarrow \mathbb{Z}[\quad, E] \rightarrow \mathbb{Z}[\quad, V] \rightarrow \underline{\mathbb{Z}} \rightarrow 0
$$

is a short exact sequence of $\mathscr{O} G$-modules.

Proof. If $H$ is any finite subgroup of $G$ then the fixed set $T^{H}$ is again a tree and hence acyclic. Thus the corollary follows from Lemma 3.3 .

Lemma 3.5. Let $G$ be a group and let $B$ be a subgroup. If $B$ is of type Bredon FP then $\mathbb{Z}[, B \backslash G]$ is an $\mathscr{O} G$-module of type Bredon FP.

Proof. This follows in much the same way as the corresponding result for groups of type FP. First, there is a functor $\times_{B} G: \mathscr{O} B \rightarrow \mathscr{O} G$. This facilitates a restriction functor from the category of $\mathscr{O} G$-modules to the category of $\mathscr{O} B$-modules and the restriction functor has, in turn, a left adjoint called induction. We need to know that the induction functor is exact, that it carries finitely generated projective $\mathscr{O} B$ modules to finitely generated projective $\mathscr{O} G$-modules, and that $\operatorname{Ind}_{B}^{G} \mathbb{Z}[-, \underline{\mathbb{Z}}]_{B} \cong$ $\mathbb{Z}[-, B \backslash G]_{G}$. The lemma follows from these facts by applying induction to a finite projective resolution of the trivial $\mathscr{O} B$-module $\underline{\mathbb{Z}}$.

Details can be found in Symonds exposition [33]. For the reader's convenience we include a summary of the key steps. 
The induction functor is defined in [24,9.15] and takes the following form in our notation.

$$
\left(\operatorname{Ind}_{B}^{G} M\right)(S \backslash G)=M(-) \otimes_{\mathscr{O} B} \mathbb{Z}\left[S \backslash G,-\times_{B} G\right]_{G}
$$

where $M$ is a $\mathscr{O} B$-module, $S$ a finite subgroup of $G$ and $\otimes_{\mathscr{O} B}$ is the tensor product defined for example in [24, 9.12]. For any finite subgroup $L$ of $B$ there is a Yonedatype formula for this tensor product:

$$
M(-) \otimes_{\mathscr{O} B} \mathbb{Z}[L \backslash B,-]_{B}=M(L \backslash B) .
$$

There is a bijection

$$
\left[S \backslash G,-\times_{B} G\right]_{G} \cong \bigsqcup_{x \in(B \backslash G)^{S}}\left[S^{\left.x^{-1} \backslash B,-\right]_{B},}\right.
$$

and, taking free abelian groups on both sides, this gives rise to an isomorphism

$$
\mathbb{Z}\left[S \backslash G,-\times_{B} G\right]_{G} \cong \bigoplus_{x \in(B \backslash G)^{S}} \mathbb{Z}\left[S^{\left.x^{-1} \backslash B,-\right]_{B}}\right.
$$

This together with the Yoneda formula above yield

$$
\left.\operatorname{Ind}_{B}^{G} M\right)(S \backslash G)=\bigoplus_{x \in(B \backslash G)^{S}} M\left(S^{x^{-1}} \backslash B\right) .
$$

This formula is used in [33] to define induction. Note that as exactness means exactness upon evaluation from the formula above one deduces that induction is exact [33, 2.9]. And in the particular case when $M=\mathbb{Z}[\quad, L \backslash B]$ we get

$$
\operatorname{Ind}_{B}^{G} \mathbb{Z}[\quad, L \backslash B]_{B}=\mathbb{Z}[\quad, L \backslash G]_{G} .
$$

This implies that $\operatorname{Ind}_{B}^{G}$ takes finitely generated free $\mathscr{O} B$-modules to finitely generated free $\mathscr{O} G$-modules (see [33, Lemma 2.9]), (which is not a surprise as the restriction functor is exact) and also implies that $\operatorname{Ind}_{B}^{G} \underline{\mathbb{Z}}=\mathbb{Z}[\quad, B \backslash G]_{G}$ (this is [33, Lemma 2.7]).

We shall work with the Grothendieck group $K_{0}(\mathscr{O} G)$ of finitely generated projective $\mathscr{O} G$-modules. If $P$ is a finitely generated projective $\mathscr{O} G$-module then we write $[P]$ for the corresponding class in the Grothendieck group. If $M$ is an $\mathscr{O} G$ module of type Bredon FP then we write $[M]$ for the element $\sum_{i \geq 0}(-1)^{i}\left[P_{i}\right]$ in $K_{0}(\mathscr{O} G)$ where $P_{*} \rightarrow M$ is any choice of finite projective resolution of $M$ over $\mathscr{O} G$. An application of Schanuel's lemma shows that $[M]$ is well-defined.

Lemma 3.6. Let $G$ be a group of type Bredon FP. If $[\underline{\mathbb{Z}}]=0$ in $K_{0}(\mathscr{O} G)$ then $G$ is of type Bredon FL.

Proof. This is a special case of [24, Theorem 11.2a]. More generally one can work with the image of $[\underline{\mathbb{Z}}]$ in $\widetilde{K}_{0}(\mathscr{O} G)$ the quotient of the Grothedieck group modulo the subgroup generated by classes of Bredon free modules and it is sufficient to check vanishing there.

Lemma 3.7. Let $0 \rightarrow A \rightarrow B \rightarrow C \rightarrow 0$ be a short exact sequence of modules of type Bredon FP over $\mathscr{O} G$. Then the equation $[C]=[B]-[A]$ holds in the Grothendieck group $K_{0}(\mathscr{O} G)$. 
Proof. Choose finite projective resolutions $P_{*} \rightarrow A$ and $Q_{*} \rightarrow C$. Then the horseshoe lemma can be used to construct a resolution of $B$ in which the $n$th projective is $P_{n} \oplus Q_{n}$. Now it is immediate that $[B]=[A]+[C]$ and the result follows.

Corollary 3.8. Let $G=B *_{B, t}$ be an ascending $H N N$-extension in which $B$ is of type Bredon FP. Then $G$ is of type Bredon FL.

Proof. There is an action of $G$ on a tree with one orbit of edges so that the $G$ sets of vertices and edges are both isomorphic to the transitive $G$-set $B \backslash G$. Using Corollary 3.4 this gives rise to a short exact sequence of $\mathscr{O} G$-modules

$$
0 \rightarrow \mathbb{Z}[\quad, B \backslash G] \rightarrow \mathbb{Z}[\quad, B \backslash G] \rightarrow \underline{\mathbb{Z}} \rightarrow 0 .
$$

Lemma 3.5 shows that the module $\mathbb{Z}[, B \backslash G]$ which appears here is of type Bredon FP. By Lemma 3.7 we have $[\underline{\mathbb{Z}}]=0$. Hence by Lemma 3.6, $G$ is of type Bredon FL.

Note that the results above also imply that if $G$ acts on a tree of finite type with stabilizers which are of type Bredon FL then $G$ itself is of type Bredon FL.

\section{Proof of THEOREM 1.1 ( $\mathrm{x}) \Rightarrow$ (i)}

Lemma 4.1. Let $G$ be a group of type Bredon FL. Then

(i) the Weyl group $H \backslash N_{G}(H)$ associated to any finite subgroup $H$ of $G$ is of type $\mathrm{FP}_{\infty}$ and in particular it is finitely generated; and

(ii) $G$ is of type Bredon $\mathrm{F}$ if and only if all Weyl groups of finite subgroups of $G$ are finitely presented.

Proof. Part (i) follows from Lemma 3.1. Lück showed [25, Theorem 5.1] that a group admits a finitely dominated model for $E G$ if and only if it satisfies the conditions in (i) and all Weyl groups of finite subgroups are finitely presented. Since $G$ is of type Bredon FL the equivariant finiteness obstruction vanishes and (ii) follows.

Lemma 4.2. Let $L=B *_{B, t}$ be an ascending $H N N$-extension over a finitely generated virtually nilpotent base $B$. Then $L$ is of type Bredon $\mathrm{F}$.

Proof. The finitely generated virtually nilpotent group $B$ has type Bredon $\mathrm{F}$ and it follows from Corollary 3.8 that $L$ has type Bredon FL. By Lemma 4.1 (i), the Weyl groups of finite subgroups of $L$ are finitely generated. As explained in [8], $L$ is a coherent group meaning that all finitely generated subgroups are finitely presented. Therefore all normalizers of finite subgroups, and the corresponding Weyl groups, of $L$ are finitely presented and $L$ is of type F by Lemma 4.1 (ii).

Proof of Theorem $1.1(x) \Rightarrow(i)$. If $G$ is polycyclic-by-finite it is of type Bredon F, which can be seen by an induction on the Hirsch length of the group, see [26, Example 5.26]. Therefore we suppose that $G$ satisfies the second part of the statement. Any finite subgroup of $G$ is therefore contained in a subgroup $L$ which is a finite index overgroup of the group $K$. Moreoever, $L$ is an ascending HNN-extension over a finitely generated virtually nilpotent group $B$ and so is of type Bredon $\mathrm{F}$ by Lemma 4.2. Also, $G / K$ is a Euclidean crystallographic group and so is of type F. The following result of Lück may therefore be used to complete the proof. 
Proposition 4.3 ([25], Theorem 3.2). Let $G$ be a group with a normal subgroup $K$ such that $G / K$ is of type Bredon F. Suppose that for each subgroup $L \supseteq K$ of $G$ such that $L / K$ is finite, $L$ is a type Bredon $\mathrm{F}$. Then $G$ is of type Bredon $\mathrm{F}$.

\section{APPENDIX: THE NON-REVERSIBILITY OF THE IMPLICATONS IN Figure 1}

$\alpha$ The celebrated paper [5] of Bestvina and Brady includes constructions for groups which are of type $\mathrm{FP}_{2}$ but not finitely presented, showing that $v$ is not a reversible implication. Such a group can be chosen to be of type FL and then it illustrates the non-reversibility of the implications marked $\alpha$. It remains conceivable that soluble groups of type $\mathrm{FP}_{2}$ are always finitely presented. The first major positive result in this direction was that of Bieri and Strebel which established the equivalence of type $\mathrm{FP}_{2}$ and finite presentability for metabelian groups using their method of invariants on the valuation sphere, [9].

$\beta$ Thompson's group $F$ shown to be of type $\mathrm{FP}_{\infty}$ by Brown and Geoghegan [14] has infinite cohomological dimension and is also torsion-free. It is a group of type Bredon $\mathrm{FP}_{\infty}$ and not Bredon FP as well as being a group of type $\mathrm{FP}_{\infty}$ and not FP.

$\gamma$ Any non-trivial finite group shows that the implications marked $\gamma$ are not reversible. A perhaps more significant observation is that if $X$ denotes any of the types F, FL, FP then "type Bredon X" neither implies nor is implied by "virtually of type X". In one direction, Raghunathan gave examples [30, 31] of groups which are not residually torsion-free but which are patently of type Bredon F. Conversely there are Leary-Nucinkis examples described in the next item which are virtually of type $F$ but fail all the type Bredon X conditions.

$\delta$ Leary-Nucinkis [23] have constructed groups which are virtually of type $\mathrm{F}$ and hence of type $\mathrm{FP}_{\infty}$ but which are not of type Bredon $\mathrm{FP}_{1}$.

$\varepsilon$ Many examples are known of groups which are of type $\mathrm{FP}_{2}$ or finitely presented but not $\mathrm{FP}_{\infty}$. There are also examples for each $n$ of groups which are of type $\mathrm{FP}_{n}$ but not of type $\mathrm{FP}_{n+1}$. Some can be found in the work [2, 4] of Abramenko and Behr. Further examples can be found in work of Abels and Brown [1, 13] There are many other such examples in the literature, too numerous to mention in detail here.

\section{REFERENCES}

[1] H. Abels and K. S. Brown. Finiteness properties of solvable $S$-arithmetic groups: an example. In Proceedings of the Northwestern conference on cohomology of groups (Evanston, Ill., 1985), volume 44, pages 77-83, 1987.

[2] P. Abramenko. Finiteness properties of Chevalley groups over $\mathbf{F}_{q}[t]$. Israel J. Math., 87(13):203-223, 1994.

[3] G. Baumslag and R. Bieri. Constructable solvable groups. Math. Z., 151(3):249-257, 1976.

[4] H. Behr. Higher finiteness properties of $S$-arithmetic groups in the function field case I. In Groups: topological, combinatorial and arithmetic aspects, volume 311 of London Math. Soc. Lecture Note Ser., pages 27-42. Cambridge Univ. Press, Cambridge, 2004.

[5] M. Bestvina and N. Brady. Morse theory and finiteness properties of groups. Invent. Math., 129(3):445-470, 1997.

[6] R. Bieri. Homological dimension of discrete groups. Queen Mary College Mathematical Notes. Queen Mary College Department of Pure Mathematics, London, second edition, 1981. 
[7] R. Bieri, W. D. Neumann, and R. Strebel. A geometric invariant of discrete groups. Invent. Math., 90(3):451-477, 1987.

[8] R. Bieri and R. Strebel. Soluble groups with coherent group rings. In Homological group theory (Proc. Sympos., Durham, 1977), volume 36 of London Math. Soc. Lecture Note Ser., pages 235-240. Cambridge Univ. Press, Cambridge, 1979.

[9] R. Bieri and R. Strebel. Valuations and finitely presented metabelian groups. Proc. London Math. Soc. (3), 41(3):439-464, 1980.

[10] R. Bieri and R. Strebel. A geometric invariant for modules over an abelian group. J. Reine Angew. Math., 322:170-189, 1981.

[11] R. Bieri and R. Strebel. A geometric invariant for nilpotent-by-abelian-by-finite groups. J. Pure Appl. Algebra, 25(1):1-20, 1982.

[12] N. Brady, I. J. Leary, and B. E. A. Nucinkis. On algebraic and geometric dimensions for groups with torsion. J. London Math. Soc. (2), 64(2):489-500, 2001.

[13] K. S. Brown. Finiteness properties of groups. In Proceedings of the Northwestern conference on cohomology of groups (Evanston, Ill., 1985), volume 44, pages 45-75, 1987.

[14] K. S. Brown and R. Geoghegan. An infinite-dimensional torsion-free $\mathrm{FP}_{\infty}$ group. Invent. Math., 77(2):367-381, 1984.

[15] D. Gildenhuys. Classification of soluble groups of cohomological dimension two. Math. Z., 166(1):21-25, 1979.

[16] D. Gildenhuys and R. Strebel. On the cohomological dimension of soluble groups. Canad. Math. Bull., 24(4):385-392, 1981.

[17] D. Gildenhuys and R. Strebel. On the cohomology of soluble groups. II. J. Pure Appl. Algebra, 26(3):293-323, 1982.

[18] K. W. Gruenberg. Cohomological topics in group theory. Lecture Notes in Mathematics, Vol. 143. Springer-Verlag, Berlin, 1970.

[19] J. A. Hillman and P. A. Linnell. Elementary amenable groups of finite Hirsch length are locallyfinite by virtually-solvable. J. Austral. Math. Soc. Ser. A, 52(2):237-241, 1992.

[20] P. H. Kropholler. Cohomological dimension of soluble groups. J. Pure Appl. Algebra, 43(3):281-287, 1986.

[21] P. H. Kropholler. On groups of type (FP) $)_{\infty}$. J. Pure Appl. Algebra, 90(1):55-67, 1993.

[22] P. H. Kropholler. Soluble groups of type (FP) $)_{\infty}$ have finite torsion-free rank. Bull. London Math. Soc., 25(6):558-566, 1993.

[23] I. J. Leary and B. E. A. Nucinkis. Some groups of type VF. Invent. Math., 151(1):135-165, 2003.

[24] W. Lück. Transformation groups and algebraic K-theory, volume 1408 of Lecture Notes in Mathematics. Springer-Verlag, Berlin, 1989., Mathematica Gottingensis.

[25] W. Lück. The type of the classifying space for a family of subgroups. J. Pure Appl. Algebra, 149(2):177-203, 2000.

[26] W. Lück. Survey on classifying spaces for families of subgroups. In Infinite groups: geometric, combinatorial and dynamical aspects, volume 248 of Progr. Math., pages 269-322. Birkhäuser, Basel, 2005.

[27] C. Martínez-Pérez and B. E. A. Nucinkis. Virtually soluble groups of type $\mathrm{FP}_{\infty}$. preprint, 12 pp., 2007.

[28] B. E. A. Nucinkis. Cohomology relative to a $G$-set and finiteness conditions. Topology Appl., 92(2):153-171, 1999.

[29] B. E. A. Nucinkis. Is there an easy algebraic characterisation of universal proper $G$-spaces? Manuscripta Math., 102(3):335-345, 2000.

[30] M. S. Raghunathan. Torsion in cocompact lattices in coverings of $\operatorname{Spin}(2, n)$. Math. Ann., 266(4):403-419, 1984.

[31] M. S. Raghunathan. Corrigendum: "Torsion in cocompact lattices in coverings of $\operatorname{Spin}(2, n)$ " [Math. Ann. 266 (1984), no. 4, 403-419; MR0735524 (86k:22027)]. Math. Ann., 303(3):575578, 1995.

[32] U. Stammbach. On the weak homological dimension of the group algebra of solvable groups. J. London Math. Soc. (2), 2:567-570, 1970.

[33] P. Symonds. The Bredon cohomology of subgroup complexes. J. Pure Appl. Algebra, 199(13):261-298, 2005. 
[34] T. tom Dieck. Transformation groups, volume 8 of de Gruyter Studies in Mathematics. Walter de Gruyter \& Co., Berlin, 1987.

P. H. Kropholler, Department of Mathematics, University of Glasgow, 15 UniVERSITY GARDENS, GLASGOW G12 8QW, SCOTLAND, UNITEd KINGDOM

E-mail address: p.h.kropholler@maths.gla.ac.uk

C. Martínez-Pérez Departamento de Matemáticas, Universidad de Zaragoza, 50009 ZARAGOZA, SPAIN

E-mail address: conmar@unizar.es

B. E. A. Nucinkis, School of Mathematics, University of Southampton, SouthampTON, SO17 1BJ, ENGLAND, UNITED KINGDOM

E-mail address: bean@soton.ac.uk 